\title{
Procedimientos y resultados de calidad en los servicios informativo-documentales
}

\author{
María Pinto
}

Universidad de Granada

\subsection{Resumen}

El objetivo general de la máxima calidad, o excelencia, en los servicios informativo/documentales se consigue mediante una política de Gestión de Calidad Total cuyos procedimientos son la planificación estratégica (que incluye el liderazgo) y la gestión de recursos (humanos, financieros, materiales, informativos) y de procesos (administrativos, analíticos, técnicos), lo que proporciona un determinado nivel de satisfacción a usuarios, personal, empresarios y ciudadanos en general, estado que retroalimenta el proceso en una serie ininterrumpida de ciclos de mejora. (Author)

Palabras clave: Gestión Calidad. Servicio de Información. Métodos de Calidad.

\subsection{Abstract}

The general objective of maximum quality, or excellence, in the informative/ documentary services is pursued through a Management policy of Total Quality whose procedures are the strategic planning (that includes the leadership) and the management of resources (human, financial, material, informative) and of processes (administrative management, analitical, technical), that provides a satisfaction level to clients, staff, managers and citizens; state that feedbacks the process in a series of uninterrupted improvement cycles. (Autor)

Keywords: Management Quality. Information Service. Methods of Quality.

\section{Introducción}

El principio clásico en economía fundamentado en la escasez o limitación de los recursos naturales en un mercado de creciente demanda puede ser extrapolado al mercado de la información/documentación, un bien aparentemente abundante pero cuyos mecanismos de selección y filtración a veces no son suficientes, y por consiguiente, no satisfacen las expectativas del cliente de los servicios. Sus escasos niveles de Calidad, entendida como grado de satisfacción alcanzado por 
los usuarios, hacen que ésta, un bien escaso o raro, adquiera altos niveles de cotización en un expansivo mercado. No resulta extraño que la Calidad sea un asunto de actualidad que provoca el interés en todo tipo de empresas informativo/documentales. La Calidad es la inversión más inteligente a medio y largo plazo que un directivo puede hacer (Bouthillier, 1992).

En el ámbito de la documentación, la década de los noventa se caracteriza por un notable crecimiento de la producción, un aumento del coste de los documentos y de los recursos, y una progresiva disminución de las partidas presupuestarias, con el consiguiente empobrecimiento empresarial. De ahí que el reto de las unidades de Información del nuevo milenio sea responder, dentro de estas limitaciones reales, a las necesidades y expectativas de los distintos segmentos de usuarios/clientes, mejorando servicios y prestaciones sin valor económico añadido y adoptando modelos más dinámicos e integrados de organización, basados en los conceptos de competencia, eficacia, eficiencia y rendimiento. Hay que concebir las actividades documentales como acciones empresariales y de gestión, entendiendo por tal la utilización óptima de los medios disponibles para satisfacer unos objetivos prefijados. Para ello, desde el prisma de la Calidad Total, el gestor de información deberá incidir en todas las variables que permiten el funcionamiento de la unidad de información, incluidos proveedores, necesidades de información, especificaciones técnicas, procesos, productos, servicios, usuarios, tiempo, costes ...

\section{El paradigma emergente de la calidad}

Al examinar el concepto de la Calidad entre los responsables de empresas de servicio, todavía nos encontramos con tecnicismos que basan la calidad exclusivamente en las cualidades de los productos, tales como conformidad con especificaciones, taso cero defectos..., sin hacer apenas consideraciones a factores de servicio que acompañan al producto.

En los últimos años la gestión de calidad se ha ampliado a los servicios, y al centro de convergencia e inmediato contacto entre el proveedor del servicio y el cliente. El hecho de que los requisitos del cliente puedan ser modificados como parte de suministro del servicio es un desafío y una oportunidad. Los clientes son participes del servicio, puesto que pueden alterar el producto suministrado y el modo en que esto se hace en respuesta a una determinada situación, están involucrados en el servicio y su suministro. La calidad necesita considerarse en términos de enganchar y apoderar al participante (Brophy; Coulling, 1997).

El servicio informativo-documental tiene la particularidad de ser ofrecido y consumido simultáneamente, y por ello el cliente no tiene la posibilidad de probar el servicio antes de adquirirlo, convirtiéndose no solo en destinatario final 
que paga el servicio, sino también en juez del rendimiento de quienes lo prestan. Es más difícil gestionar la calidad de un servicio que la de un producto pues viene definida en función de la percepción del cliente sobre el servicio que recibe, que alcanzará la calidad necesaria cuando reúna o supere las expectativas de los clientes sobre cómo se deben prestar esos servicios.

Por ello se hace imprescindible la implantación de una determinada infraestructura conceptual que favorezca la adopción en el Servicio de Información/Documentación del paradigma de la Calidad, y más concretamente de la Gestión de Calidad Total — Total Quality Management (TQM)—, una aproximación sistemática y holística a los problemas de gestión de las organizaciones cuyo objetivo principal es el logro de un estado de máxima satisfacción generalizada: de clientes, de empleados, de empresarios y de la comunidad en general. Teniendo en cuenta este objetivo de Calidad Total, o Global, mas que de calidad en singular conviene hablar de calidades, pues la Calidad Total depende de una serie de calidades parciales que contribuyen a ese objetivo de máxima satisfacción generalizada previamente establecido. En una línea de investigación que trata de compatibilizar las Normas ISO 9000 con los distintos planteamientos teóricos de las instituciones que trabajan para la Calidad Total (Deming, Baldridge, EFQM, ...), el objetivo principal de este artículo es provocar en gestores, empleados y usuarios de los servicios de información una nueva conciencia sobre como concebir de un modo amigable un instrumento metodológico de Planificación, autoevaluación y mejora de los niveles de Calidad.

Este estado de máxima satisfacción generalizada, muy asimilable a lo que en la actualidad se entiende por excelencia, debe aplicarse a todos los individuos y entidades que, directa o indirectamente, tienen relación con el servicio de información y es mejor comprendido como una reacción emocional al servicio/producto suministrado, a pesar de las muchas investigaciones empíricas que tienden a las medidas no afectivas, basadas mas en la satisfacción de determinadas necesidades que en la satisfacción de las personas. En cualquier caso el ingrediente emocional resulta fundamental para el estudio de los aspectos relacionados con la Calidad: la satisfacción es una emoción y una reacción en la que las expectativas juegan un papel crucial, de tal modo que una discusión sobre la satisfacción y su formación debe también incluir una discusión sobre las expectativas, sus tipos y su formación (Applegate, 1994). Ni que decir tiene que cualquier iniciativa TQM no tiene limitación en el espacio, pues debe ser generalizada, ni tampoco en el tiempo, pues su objetivo de máxima satisfacción jamas se consigue plenamente. De ahí el proceso de mejora continua que preside el concepto TQM.

Por motivos funcionales, y sobre todo expositivos, distinguimos entre procedimientos y resultados de la Calidad (Fig. 1). La secuencia comienza con el planteamiento de unos objetivos globales que, a falta de ser concretados en cada

Scire. $5: 1$ (en.-jun. 1999) 11-25. 


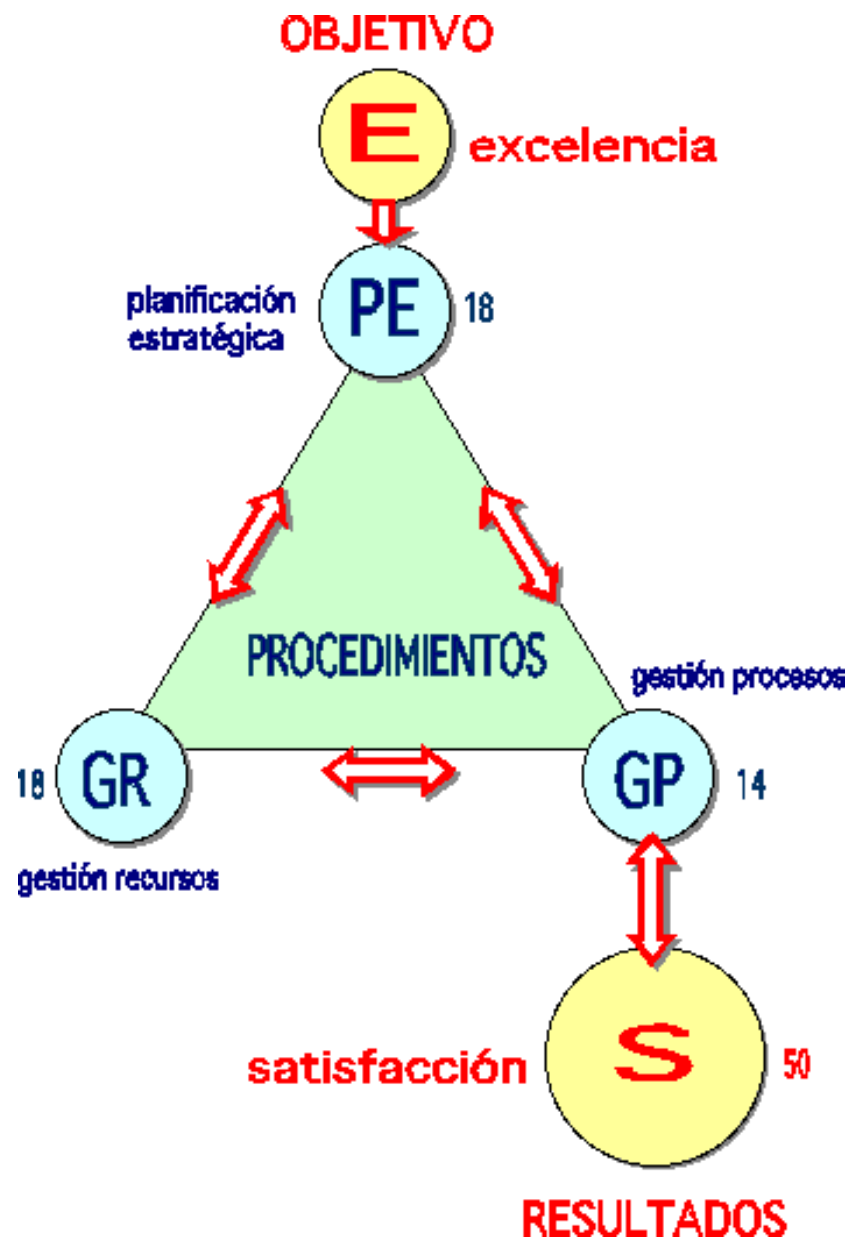

Fig. 1. Procedimientos y resultados de la calidad

caso particular, hemos resumido en ese estado ideal de máxima satisfacción generalizada, o excelencia. El despliegue de tales objetivos, o expectativas, se lleva a cabo mediante una serie de procedimientos encadenados, o enlazados, a los que por idénticas razones hemos agrupado en Estrategias, Gestión de recursos y Gestión de procesos. La interdependencia de estos tres compartimentos de la Calidad es absoluta, y su importancia relativa es muy similar como puede comprobarse en la valoración que sobre una puntuación total de 100 hemos asignado 
a cada uno de ellos. El Rendimiento Global de acuerdo con estos criterios de planificación, autoevaluación o mejora se expresará mediante la suma de la puntuación asignada a cada compartimento, que no son estancos sino entrelazados a modo de "vasos comunicantes" permitiendo a su través el flujo de la Calidad del Servicio. Mientras los objetivos indican lo que el Servicio de información pretende, los procedimientos nos dan una idea precisa del modo en que se está operando para alcanzar tales logros. Los resultados, plasmados en un determinado nivel de satisfacción, nos indican los logros pero al mismo tiempo señalan los retos por alcanzar, de tal manera que, en lugar de tratarse de un final de trayecto, nos hallamos ante el comienzo de un nuevo ciclo de calidad que, retroalimentado desde estos resultados, inicia de nuevo los procesos en pos de ese grado de excelencia inalcanzable. La importancia de los resultados como punto de arranque del nuevo ciclo es tal que se le ha asignado una puntuación de 50, equiparándose al total de los procedimientos. Se da de este modo la aparente paradoja de que la consecuencia es tan importante como las causas. Y lo es, porque a su vez se transforma en causa del ciclo siguiente de calidad en una serie ininterrumpida.

\section{Procedimientos para la calidad}

\subsection{Planificación Estratégica}

Un factor clave, sobre todo porque se sitúa en el origen del organigrama de la Calidad, es el establecimiento de una política de planificación estratégica clara y concisa, que defina la Misión del Servicio de información, es decir el papel global del centro como empresa de producción y prestación de servicios informativos documentales. La Misión debe desplegarse en Metas, o amplia declaración de fines; Objetivos específicos de cada meta; y Estrategias, o acciones concretas necesarias para lograr esas metas y objetivos previamente establecidos. El Servicio, en consonancia con una Misión perfectamente definida, deberá elaborar un Plan Estratégico de Calidad (Misión, Metas, Objetivos, Estrategias) que conlleve el correspondiente cambio cultural en todos los ámbitos del centro, adoptando la mejora continua como actitud y el enfoque al cliente/usuario como estrategia. De esta manera la Calidad (Senlle, 1996) pasa de ser un costo añadido un valor añadido al producto o servicio suministrado, convirtiéndose en un factor de ahorro que asegura la competitividad y supervivencia de la organización.

La Planificación Estratégica se plasmará en el Reglamento del Servicio, marco normativo y elemento de control útil para valorar los objetivos y las metas. Deberá basarse en el concepto de Calidad Total, fundamentarse en datos relevantes, comunicarse a lo largo y ancho de la organización, y revisarse y mejorarse continuamente (Johannsen, 1995). Asimismo dicha planificación quedará

Scire. $5: 1$ (en.-jun. 1999) 11-25. 
reflejada en el Manual de Calidad que contemplará el compromiso firmado de la organización con la Calidad, la relación de responsables de mantenimiento del programa así como la descripción de los procedimientos necesarios para conseguir el objetivo final de la Calidad.

Este es el mejor modo de relacionar e integrar el Servicio de información en un entorno cambiante, con sus limitaciones y posibilidades correspondientes. Un problema importante en la planificación estratégica de estos servicios se origina al valorar los datos de partida, base para las posteriores tomas de decisiones y establecimiento de mejoras, pues lo normal es que se registren regularmente los datos relativos a instalaciones, equipamiento, presupuesto, actividades, colecciones y procesos. Pero estos datos cuantitativos, aunque son sumamente importantes, obvian aspectos tan relevantes para el servicio como son el rendimiento de las colecciones o la satisfacción de los usuarios por ejemplo. En cualquier caso, la participación del personal en la definición de la política estratégica del centro es un asunto fundamental.

Por su parte el liderazgo supone el comportamiento del individuo o grupo responsable de la dirección en pos de una cultura TQM que "salpique" a todos los "rincones" del Servicio. El líder (persona o equipo) debe inspirar, apoyar y promover tal cultura, demostrando visiblemente su compromiso y apoyando la implicación de todos los integrantes de la organización en la mejora continua. Se comprometerá con clientes, proveedores y otras organizaciones externas, escuchándoles y facilitando la innovación (Austin, 1986), reconocerá los esfuerzos y logros del personal y articulará mecanismos de relación fluida entre los responsables del servicio y sus componentes (creación de órganos, desarrollo de métodos de comunicación apropiados, trabajo en equipo, trabajo por objetivos), estimulando las relaciones humanas, auténtico resorte para la implantación de Sistemas de Calidad.

\subsection{Gestión de los Recursos}

Este apartado de recursos se refiere a cómo la organización gestiona, planifica, utiliza y conserva de un modo eficaz y eficiente los recursos, tanto financieros, informativos, humanos como materiales.

\subsubsection{Recursos Financieros}

Se debe potenciar la autonomía del Servicio de información como un único centro de gasto, mediante un Plan de Acción Económica que tenga en cuenta las necesidades reales y potenciales.

\subsubsection{Recursos Informativos}

Se promocionarán los recursos informativos mediante la definición de un programa integrador que desarrolle la colección, teniendo en cuenta los criterios 
del profesional y las expectativas /necesidades de los usuarios. Además se fomentaran y aprovecharan los recursos informativos en red y se diseñarán programas de expurgo, reubicación y preservación de los documentos.

\subsubsection{Recursos Humanos}

Como en toda industria de servicios, la prioridad de los recursos humanos es un hecho incuestionable, pues la persona (proveedor, personal, cliente) es el recurso clave del Servicio, de manera que determinadas carencias en los recursos de tipo financiero, informativo o material pueden ser superadas mediante unos recursos humanos excelentes. Por el contrario, un Servicio con excelentes recursos financieros, informativos y materiales puede hacer aguas por su falta de Calidad en lo que a recursos humanos se refiere. El capital humano es el recurso estratégico fundamental de una organización (Blanco, 1998). Por ello, hay que darle poder, "apoderarlo", para conseguir poder.

Es importante reconocer los esfuerzos y logros del personal, potenciando programas de promoción y de crecimiento de plantilla, de manera que sea adecuada en relación con el número de usuarios, puntos de servicio, horas de funcionamiento y áreas de trabajo del centro. Se diseñarán planes de formación continua que proporcionen al personal la formación adecuada no solo en el saber hacer sino también y sobre todo en el saber ser, potenciando actitudes, motivaciones, comportamientos, percepciones y relaciones, e impulsando el concepto de autonomía, responsabilidad y compromiso entre sus miembros, para que participen desde los distintos puestos de trabajo en aras de un alto grado de excelencia.

El Servicio de Información y Documentación deberá desarrollar todo el potencial de su personal en aras de una mejora continua de la organización. Para ello es importante que:

- Se planifiquen y mejoren los recursos humanos.

- Se mantengan y desarrollen las capacidades humanas mediante formación y entrenamiento.

- El personal esté de acuerdo con los objetivos y periódicamente se revisen rendimientos.

- Todo el personal esté implicado, facultado y reconocido en el proceso de mejora continua.

- El personal y la organización tengan un dialogo efectivo.

Por consiguiente la definición de una Política de Personal clara establecerá el grado de formación apropiado, la adecuada asignación de funciones y niveles de responsabilidad y el desarrollo de planes de formación integrales acordes con la política global del centro. Y puesto que la Calidad del Servicio depende también 
de la forma en que los empleados desarrollan sus tareas, se analizará regularmente el rendimiento del personal.

\subsubsection{Recursos Materiales}

Se cuidará la planificación espacial y funcional de las instalaciones a medio y largo plazo teniendo en cuenta las sugerencias de documentalistas y usuarios. También se velará por los niveles de equipamiento y por la mejora constante de los recursos informáticos.

\subsection{Gestión de los Procesos}

La importancia superlativa que tienen los Procesos en el ámbito de los agentes de la Calidad es una realidad incuestionable, asumiéndose la idea de que la clave de la Calidad radica fundamentalmente en los procesos. Este comentario es perfectamente válido para el entorno informativo/documental en que nos desenvolvemos, en virtud de las propiedades específicas de los procesos, productos y servicios correspondientes. Un Servicio de información y documentación que aspire a mejorar sus niveles de Calidad debe:

- Identificar los procesos que son clave para el éxito empresarial, teniendo en cuenta las necesidades y expectativas del cliente.

- Gestionarlos sistemáticamente.

- Revisarlos y fijar objetivos de mejora.

- Mejorarlos utilizando la innovación y la creatividad.

- Evaluar los beneficios.

En un contexto TQM hablar de procesos implica tener que aludir forzosamente no solo a productos sino también a servicios, pues no existe una frontera claramente delimitada entre estos tres elementos de los sistemas de producción y servicio, frontera que es mucho más sutil y a veces inapreciable en el caso de los sistemas informativo/documentales. A grandes rasgos podemos afirmar que mientras un proceso es una actividad de valor añadido, un producto es el resultado acabado de un proceso y un servicio es un proceso que consta de una serie de actividades regidas por la conducta y actitudes de las personas implicadas. En el entorno informativo la prestación de un servicio es considerada como parte de un proceso con participación del cliente, que puede ser visto como un co-productor del Servicio. En cualquier caso, y admitiendo que procesos, productos y servicios son realidades distintas que se entremezclan y confunden en virtud de la habitual dependencia del servicio con respecto al producto en que se basa, hemos de reconocer que gozan de bastante autonomía funcional, sobre todo a la hora de controlar su Calidad. Si tenemos en cuenta que todo Servicio documental combina la producción de bienes (catálogos, índices..) con la prestación de servicios, com-

Scire. $5: 1$ (en.-jun. 1999) 11-25. 
prenderemos la necesidad de aplicar Programas de Calidad no solo como instrumento de diseño y sistema de control para que el producto fabricado cumpla unas especificaciones técnicas precisas sino también como método de actuación en las relaciones profesional/cliente, pues no olvidemos que los servicios se prestan con el ingrediente fundamental de las diferencias humanas individuales.

En todo caso es incuestionable la ventaja de incorporar al desarrollo de los procesos la voz del cliente, para lo cual se utiliza un procedimiento conocido como QFD (Despliegue de la Función Calidad), que despliega las expectativas del cliente para transformarlas de un modo matricial en características del producto/servicio, manifestándose también la prioridad que los clientes dan a algunas de estas características. QFD supone el despliegue objetivo y detallado de las funciones u operaciones que conforman sistemáticamente la calidad de un producto (Mizuno ; Akao, 1978).

Restringidos al entorno informativo, hemos de destacar la importancia de los siguientes tipos de procesos:

\subsubsection{Procesos de Gestión Administrativa}

Aglutinan todos los aspectos relacionados con el aprovisionamiento y suministro de documentos a la unidad de información, así como las tareas referentes a la tramitación de pedidos, verificación de facturas, reclamaciones a proveedores, procesos en los que el Servicio actúa fundamentalmente como cliente. De cara a su optimización sería conveniente la existencia de un negociado que permitiera centralizar las tareas de administración y suministro, con el consiguiente ahorro de tiempo, normalización del material, previsión de necesidades y reparto de los recursos. En definitiva, se trataría de establecer un Sistema de Calidad de Suministradores (Muñoz; Sala, 1998)

\subsubsection{Procesos Técnicos}

Abarcan el conjunto de actividades relacionadas con el tratamiento del documento y la puesta a punto mediante el uso de los canales de circulación apropiados. Destacaremos el proceso de adquisición o selección de documentos, que exige una buena planificación y unos conocimientos adecuados para integrar la voz del experto, del documentalista y del usuario, y las operaciones de catalogación, análisis y representación de la información con el fin de crear instrumentos accesibles y normalizados. Será de gran utilidad la elaboración de un Manual de Procedimientos Técnicos, preciso y detallado, que describa la metodología de aplicación de los distintos aspectos de las Reglas de Catalogación, los procedimientos y modelos de análisis y representación de la información significativa, incidiendo en los niveles de detalle, estilo para la introducción de datos, grado de automatización de estos procesos internos... 
De entre los posibles atributos de Calidad en los procesos documentales, sobresalen la precisión en la recogida de los datos, la legibilidad, el tiempo y esfuerzo empleado en el proceso, la pertinencia y representatividad de la información, la ergonomía y visualización ...

\subsubsection{Procesos de difusión y acceso a la información}

Son procesos relacionados con el aspecto funcional del servicio, esto es el acceso a los fondos y la prestación de servicios a los usuarios/clientes. La gestión del servicio de préstamo, manifestado en el proceso pregunta - respuesta es de naturaleza cíclica pues empieza con el acceso al servicio, la concreción de las necesidades de información y su materialización para terminar con la prestación y los resultados del servicio; e interactiva, al suponer una transacción de información personalizada entre documentalista y cliente. También sería de utilidad la elaboración de un Manual de Procedimiento de Uso con las recomendaciones necesarias para el conocimiento, manejo y aprovechamiento de los recursos informativos.

\section{Resultados de calidad}

\subsection{Satisfacción del Cliente/Usuario}

La satisfacción del cliente es el auténtico núcleo o motor de la Calidad, su origen y también su destino final y definitivo, su razón de ser más universal, pudiéndose asegurar que las más diversas propuestas sobre Calidad surgidas a lo largo y ancho de nuestra geografía occidental tienen un punto de encuentro o convergencia en la satisfacción del usuario/cliente de las organizaciones. El mayor patrimonio del Servicio es su clientela, pues sin ella lo demás no tiene sentido.

Para poder incidir sobre el Grado de Satisfacción de nuestros Clientes/Usuarios es necesario conocer no solo la opinión que tienen de los productos y servicios suministrados, sino también las medidas adicionales que se toman en relación con la satisfacción de tales clientes. Un buen servicio a los usuarios/clientes es un resorte clave en la planificación estratégica, pues está ligado a la supervivencia y al rendimiento de organización, si bien hemos de reconocer las dificultades para lograr el equilibrio entre las necesidades del Servicio y las de sus clientes.

Ante el aumento de la competitividad y globalización de los distintos sectores de la economía, la Unión Europea está trabajando en el ámbito institucional para desarrollar el Indice Europeo de Satisfacción del Cliente, con el fin de facilitar a las empresas instrumentos para conocer la opinión de sus clientes, tanto en términos absolutos como relativos a la competencia del sector. Este Indice cons- 
tituirá una herramienta fundamental para la toma de decisiones sobre planificación estratégica, como nos indican los excelentes resultados obtenidos en Suecia (desde 1989), Alemania (1992), y USA (1994). Durante el presente año está previsto que los países europeos (entre ellos España) participen en un Proyecto Piloto denominado Programa EURIX para la implantación del mencionado Indice de Satisfacción del Cliente cuyo objetivo primordial es conocer su grado de satisfacción de un modo exhaustivo: a lo largo del tiempo y a distintos niveles de agrupación (consumidores, compañías de un sector...).

El análisis de la satisfacción podrá afectar al conjunto de productos del Servicio o solo a una parte según la política estratégica establecida. Así podrá referirse al servicio en sí (horario, ergonomía), a la unidad de referencia (acceso a la información, forma de transmisión de la información, calidad), a la colección (cobertura, pertinencia, agilidad en el tratamiento) y a los tipos de servicios o productos ofrecidos (préstamo, catálogos, bases de datos).

Pero el vasto trabajo desarrollado en el sector para medir la satisfacción del usuario ha sido mas bien decepcionante, en parte porque las medidas globales de satisfacción raramente apuntan el camino hacia las acciones de mejora. Por otro lado, es muy difícil cuantificar de un modo reproducible los aspectos "suaves" de la satisfacción o la relación existente entre esta y la demanda (Brophy, 1995). En cualquier caso es necesario determinar las características de las exigencias detectadas por el cliente susceptibles de ser evaluadas (ISO, 1991): cuantitativas, pues pueden ser medibles en unidades de tiempo tales como, tiempos de espera en la prestación del servicio, número de visitas para resolver un problema, tiempos de entrega de la información...; cualitativas, esto es características comparables que dependen de la forma de evaluación, como las condiciones ambientales de prestación del servicio, cortesía, amabilidad, confianza, seguridad del servicio...También, hemos de mencionar las características propias del servicio, tales como duración del proceso de prestación del servicio desde la solicitud hasta la finalización del mismo, agentes participantes, sistema de quejas y reclamaciones, nivel de fiabilidad del servicio y satisfacción mutua alcanzada, desarrollo de las relaciones interpersonales...

La medición de las características se puede llevar a cabo empleando metodología diversa en función de los aspectos tenidos en cuenta (Senlle, Vilar, 1996): estadística; temporal: tiempo de espera, demoras; por eficacia: el servicio es útil, se resuelve la necesidad de información; por actitudes: amabilidad, cortesía; por conductas observables: rapidez, lentitud, cualificación, competencia; por comparación: precio, prestaciones en relación con otras Unidades de Información similares; por grado de satisfacción del cliente: muy, satisfecho, medianamente satisfecho, insatisfecho; por economía; por los clientes que se 
pierden; por el análisis de las quejas. Cada Servicio de información empleará los elementos de medición previstos en el oportuno Plan Estratégico.

\subsection{Satisfacción del Personal}

Aunque la gestión de calidad se ha concentrado tradicionalmente en el cliente/usuario, algunos modelos de gestión, incluido el europeo en que nos apoyamos, amplia este concepto incluyendo a todas aquellas personas que tienen interés en la organización y en sus actividades. También los individuos que trabajan para una empresa (el personal) son sus clientes/usuarios y consiguientemente forman parte de la cadena de la Calidad.

Pero cuando el énfasis se pone en las nuevas tecnologías de la información, puede resultar tentador no evaluar los servicios mas que en términos de rendimiento de los medios técnicos que se ofrecen a los usuarios. No obstante, la calidad (y la cantidad) de los servicios y el rendimiento global dependen igualmente del modo en que los empleados cumplen sus tareas (Bouthillier, 1993). Sería conveniente establecer indicadores y mecanismos de medición que facilitarán información acerca del personal documentalista en aspectos relacionados con su eficacia y eficiencia, el reconocimiento de esfuerzos y méritos, su nivel de motivación, ya sea basados en criterios económicos o de promoción. Se potenciará el trabajo compartido implicando a todo el personal de la organización. Sus opiniones y su grado de satisfacción resultan fundamentales para el progreso cualitativo del servicio.

\subsection{Satisfacción del Empresario}

Aunque es un concepto bastante amplio y complejo, este resultado de la Calidad alude a lo que la unidad de información ha conseguido en relación con sus objetivos comerciales planificados, y en relación con la satisfacción de las necesidades y expectativas de todo el que tenga un objetivo financiero en la misma. Para poder incidir en este estado de satisfacción es necesario conocer los niveles de rendimiento financiero.

\subsection{Satisfacción de la Comunidad}

También se debe conocer lo que está consiguiendo el Servicio en la satisfacción de las necesidades y expectativas de la comunidad, como su aproximación a la calidad de vida, el entorno, la conservación de los recursos globales, las propias medidas internas de efectividad, así como sus relaciones con las autoridades y organismos que afectan y regulan la actividad informativo/documental. Hay que planificar, evaluar y mejorar la percepción/impacto social del Servicio en la comunidad a que pertenece. 


\section{Conclusiones}

De lo expuesto podemos inferir que las aportaciones verdaderamente interesantes de la Calidad radican en su consideración de Filosofía, de forma de ver la vida, conllevando un cambio en los valores culturales de los individuos hasta convertirse en una mística de trabajo orientada a la mejora constante. La Calidad empieza con Educación y termina con Educación. A modo de propuesta global de mejora orgánica del Servicio de información, y teniendo en cuenta las carencias y puntos críticos más frecuentes, sería necesario considerar las siguientes Oportunidades de mejora en cada uno de los Factores de Calidad considerados:

- El Servicio debe contar con su propio Documento de Política Estratégica, que diseñe el marco de actuación, la misión, las metas, los objetivos y las estrategias del servicio, y en el que se contemplen la articulación de mecanismos de relación fluida y los procedimientos de recogida de datos y estructuración funcional y de responsabilidad.

- Con objeto de fomentar el papel del líder en el organigrama funcional de la institución, se elaborará el Reglamento del Servicio, como marco normativo e instrumento de control de todos los elementos que intervienen en los complejos procesos de producción y prestación de servicios.

- Existirá un Programa de Gestión integrada de Recursos que incluya planes de formación continuada; adecuación de la plantilla; sistemas de reconocimiento de méritos; programas de gestión de recursos informativos; y una manifestación explícita sobre la necesidad de participación del gestor de información en todos los procesos.

- Se diseñarán los oportunos Manuales de Procedimientos Técnicos y de Uso, que registren de manera sistemática los procesos y productos a desarrollar, así como la prestación de servicios de ellos derivados.

- Para fomentar la satisfacción del Cliente, es necesario determinar y medir el nivel de calidad obtenido, de manera que un servicio tendrá la calidad necesaria cuando reúna o supere las expectativas de los clientes sobre cómo se debe prestar ese servicio. En este sentido se han desarrollado modelos formales que permitan mejorar la calidad del servicio (Servqual, Servperf..).

- Es necesario incrementar la satisfacción del Personal, como cliente interno que forma parte de la cadena de Calidad, mediante programas de formación integral, planes de promoción, nuevas técnicas de comunicación y de trabajo.

- Se establecerá un Plan global de Evaluación que tenga en cuenta tanto los procedimientos como los resultados de la Calidad y establezca indicadores 
de rendimiento apropiados (ISO, 1998). Se preverán planes y grupos de mejora que corrijan los puntos débiles y críticos de la unidad de información.

El desafío de todos los que de algún modo nos movemos en un entorno cambiante, con avances tecnológicos que nos imponen nuevos modos de actuación profesional y ciudadana, será mejorar nuestro Grado de Satisfacción como Usuarios, Empleados, Gestores y por supuesto Ciudadanos de a pie. No nos cabe la menor duda de que estos factores de Calidad y Excelencia, bastante introducidos en los países pioneros del mundo desarrollado, en un momento en que los procesos de cambio constituyen el auténtico motor de las organizaciones modernas, fomentarán la toma de conciencia sobre la imperiosa necesidad de una Mejora Continua en la prestación de los servicios informativos-documentales.

\section{Bibliografía}

Applegate, R. (1994). Models of satisfaction. Encyclopedia of Library and Information Science. New York : Marcel Dekker, 1994, v. 60, p. 199-227

Austin, N, Peters, T. (1986). Pasión por la Excelencia. Méjico : Lasser Press, 1986

Blanco, V. (1998). Aspectos motivadores de la Calidad en la Gestión de personas. //7 ${ }^{\circ}$ Congreso Español de la Calidad. Barcelona : AEC;Gestión 2000, 1998. p. 168-171

Bouthillier, F.(1992). La qualité totale et la qualité des services dans les bibliothéques. // Argus. 21 : 3 (Hiver, 1992). 29-31

Bouthillier, F. (1993). Services d'information et evaluation du rendement du personnel. // Argu. 22 :2 (1993). 21-34

Brophy, P. (1995). Quality Management in Libraries : Proceedings of the 1st. Northumbria International Conference : Performance measurement in Libraries and Information Services. Newcastle : University of Northumbria, 1995, p. 77-81.

Brophy, P., Coullng, K. Quality, Management for Information and Library Managers. London: Aslib, 1997

ISO: Norma ISO 9004-2, 1991. (1991). Quality managment and quality system elements. Part 2: Guidelines for services. Gènève : ISO, 1991

ISO: Norma ISO 11620-1998. (1998). Information and documentation : Library performance indicators. Gènève : ISO, 1998

Johannsen, C.G.(1995). Quality Management Principles and Methods in Library and Information Science. Theory and Practice. // Enciclopedia of Library and Information Science. New York : Marcel Dekker, 1995, v. 56, p. 318-351

Mizuno, D., Akao, Y. (1978). Quality Function Deployment. Approach for Total Quality Control. Japon : JUSE, 1978

Muñoz, J., Sala, A. (1998). La evaluación de los suministradores de servicios como una herramienta para la Gestión de Calidad de una empresa. // $7^{\circ}$ Congreso Español de la Calidad. Barcelona : AEC ; Gestión 2000, 1998, p. 77-83 
Senlle, A. (1996). Calidad total en los Servicios y en la Administración Pública. Barcelona : Gestión 2000, 1996

Senlle, A. ; Villar, J. (1996). ISO 9000 en empresas de servicios. Barcelona : Gestión 2000, 1996. 